\title{
Perfusions continues et pompes intelligentes en néonatologie : une analyse " pré-post " des modes de défaillance, de leurs effets et de leur criticité (AMDEC)
}

\author{
Emmanuelle Delage, Julien Tourel, Brigitte Martin, Aurélie Guérin, Ahmed Moussa, Annie Lacroix, \\ Denis Lebel et Jean-François Bussières
}

\section{INTRODUCTION}

$\mathrm{L}$ 'administration de médicaments par voie parentérale comporte davantage de risques que la plupart des autres voies d'administration en établissement de santét ${ }^{1-3}$. Les risques associés à l'utilisation de la voie parentérale sont accrus en néonatalogie en raison notamment des ajustements de doses selon le poids, la taille ou la surface corporelle ainsi que de la nécessité de recourir à des préparations magistrales non stériles et stériles ${ }^{4-6}$.

Il existe une variété de stratégies susceptibles de réduire les risques médicamenteux dans le circuit du médicament hospitalier, qui comprennent notamment le recours à de bonnes pratiques, la formation adéquate $\mathrm{du}$ personnel soignant et l'utilisation optimale de différentes technologies ${ }^{5-8}$. Bien que deux revues de la littérature laissent supposer que les pompes intelligentes contribuent à la réduction du risque médicamenteux en établissement de santé, les données probantes en faveur des pompes intelligentes demeurent encore limitées ${ }^{4,5,8-18}$. Il faut aussi souligner que les technologies ne sont pas sans risque et qu'elles contribuent à la création de nouveaux modes de défaillance ${ }^{13}$. Au Canada, on encourage l'utilisation des pompes intelligentes, et Agrément Canada a créé une pratique organisationnelle requise entourant la formation et l'utilisation des pompes intelligentes ${ }^{19}$.

Ces pompes dites intelligentes comportent un logiciel de gestion des doses de médicaments assorti d'une base de données qui permet d'établir pour chaque médicament une ou plusieurs concentrations permises, des limites minimales et maximales franchissables et infranchissables et des alertes ${ }^{20}$.

En gestion des risques, on recommande de mener périodiquement des analyses prospectives de processus liés à la sécurité des patients afin de mettre en ouvre des améliorations ou des changements appropriés. Parmi les méthodes d'analyse prospectives du risque, l'analyse des modes de défaillance, de leurs effets et de leur criticité (AMDEC) permet de déterminer les risques de défaillance d'un processus, de prédire les éventuels préjudices et de prioriser les actions à mener pour améliorer la sécurité du processus ${ }^{21-24}$.

En vue de sécuriser et d'améliorer l'administration parentérale des médicaments, notre centre a décidé d'implanter des pompes intelligentes afin de remplacer son parc de pompes volumétriques devenu obsolète. L'objectif de cette étude est d'évaluer, par la méthode AMDEC, le risque théorique lié au processus d'utilisation des perfusions continues, avant et après l'implantation de pompes intelligentes, au sein d'un service de néonatalogie.

\section{DESCRIPTION DE LA PRATIQUE}

Il s'agit d'une étude de type AMDEC, qui a lieu avant et après l'implantation de pompes intelligentes.

Leétude a été réalisée dans un centre hospitalier universitaire mère-enfant de 500 lits. Plus de 3460 médicaments sont inscrits à la liste locale de l'établissement, et au moins 770 sont destinés à la voie parentérale.

\section{Implantation des pompes intelligentes}

À la suite d'un appel d'offre, notre établissement a procédé à l'acquisition de 1045 pompes intelligentes (Perfusomat et Infusomat de B. Braun of Canada Ltd, Mississauga [Ontario]). Afin d'appuyer la planification, l'implantation et le soutien de ce nouveau parc de pompes, une équipe interdisciplinaire composée notamment d'un gestionnaire de projet, d'un médecin, d'infirmières, de pharmaciens et d'assistants de recherche en pharmacie, d'un technicien en informatique et en génie biomédical s'est réunie durant près de 24 mois afin de planifier 
l'intervention. Avant de réaliser l'intervention, nous avons procédé à la standardisation des concentrations de médicaments, éliminé la règle des six pour le calcul des débits de perfusion, élaboré une bibliothèque des doses limites franchissables et infranchissables, rédigé ou révisé l'ensemble des feuilles d'ordonnance prérédigées, développé des outils en ligne, formé l'ensemble des utilisateurs et assuré un soutien après l'implantation.

La méthodologie AMDEC comporte trois étapes : (1) la sélection du processus à analyser et la mise en place de l'équipe pluridisciplinaire, (2) la définition des étapes du processus et la détection des modes de défaillance et (3) la cotation des modes de défaillance.

\section{Sélection du processus à analyser et mise en place de l'équipe pluridisciplinaire}

Les étapes du processus d'analyse de l'AMDEC ont été sélectionnées par les membres de l'équipe de recherche. Afin d'obtenir différentes expertises et perspectives, nous avons constitué une équipe pluridisciplinaire composée de personnes ayant participé à l'implantation des pompes intelligentes.

\section{Définition des étapes du processus et détection des modes de défaillance}

Une réunion pluridisciplinaire a eu lieu afin de définir les étapes du processus. Les participants se sont penchés sur la détermination des modes de défaillance (c.-à-d. la forme observable du dysfonctionnement) du processus avant et après l'implantation.

\section{Cotation des modes de défaillance}

Lanalyse AMDEC repose sur la cotation de la fréquence d'occurrence (c.-à-d. probabilité d'apparition du mode de défaillance), de la gravité de l'effet (c.-à-d. gravité potentielle) et de la probabilité de non-détection de chaque mode de défaillance. Une échelle a été définie pour calculer la fréquence d'occurrence, la gravité de l'effet et la probabilité de non-détection de chaque mode de défaillance ${ }^{25}$. Enfin, nous avons établi que la nondétection de chaque mode de défaillance devait survenir dans l'heure suivant la défaillance, même si, dans la pratique, les erreurs peuvent être détectées après ce délai. Le choix s'est porté sur l'épinephrine, un médicament qui présente un risque élevé de causer un préjudice au patient en cas d'erreur, comme étalon pour la cotation des modes de défaillance ${ }^{26}$.

La cotation de la fréquence d'occurrence, de la gravité de l'effet et de la probabilité de non-détection de chaque mode de défaillance a été établie par consensus entre les membres de l'équipe pluridisciplinaire. La criticité, définie par le produit de la fréquence d'occurrence, de la gravité de l'effet et de la probabilité de non-détection, a ensuite été calculée pour chaque mode de défaillance. La criticité globale et la criticité de chaque étape du processus ont également été calculées.

\section{ÉVALUATION DE LA PRATIQUE}

La méthodologie AMDEC a été réalisée en mars 2012.

\section{Sélection du processus à analyser et mise en place de l'équipe pluridisciplinaire}

Le processus étudié concernait l'utilisation des perfusions continues de médicaments dans une unité de néonatologie de 65 lits. L'équipe pluridisciplinaire comprenait une pharmacienne responsable des soins pharmaceutiques à l'unité, un médecin et une infirmière du département de néonatologie, un pharmacien responsable de la gestion des risques et deux résidents en pharmacie.

\section{Définition des étapes du processus et détermination des modes de défaillance}

L'équipe pluridisciplinaire a choisi trois étapes du processus d'utilisation des perfusions continues, à savoir la prescription, la préparation et l'administration de doses de médicaments. L'équipe pluridisciplinaire a déterminé un total de 11 modes de défaillance avant l'implantation et de 16 modes de défaillance après l'implantation.

\section{Cotation des modes de défaillance}

L'équipe s'est réunie à deux reprises à raison de 60 minutes par séance afin de procéder à la cotation de la fréquence d'occurrence, de la gravité de l'effet et des probabilités de non-détection. Le tableau 1 présente les échelles de cotation utilisées. Les résultats des cotations de la fréquence d'occurrence, de la gravité de l'effet et de la probabilité de non-détection des modes de défaillance antérieurs et postérieurs à l'implantation sont décrits dans le tableau 2. La criticité globale a été cotée à 1064 avant l'implantation contre 814 après l'implantation.

\section{IMPLICATIONS DANS LA PRATIQUE}

\section{Diminution de la criticité globale}

Lanalyse comparative précédant et suivant l'implantation a mis en évidence une réduction globale de 250 de l'indice de criticité (c.-à-d. de 1064 à 814) du risque lié au processus d'utilisation des perfusions continues après l'implantation de pompes intelligentes. On peut donc raisonnablement penser qu'une nouvelle technologie peut contribuer à réduire les risques.

\section{Diminution de la criticité pour les étapes de prescription et de préparation}

On a également observé une réduction de la criticité aux étapes de prescription et de préparation de doses de médicaments après l'implantation. Plusieurs changements associés à l'implantation de ces pompes intelligentes peuvent expliquer cette diminution de la criticité. 
Tableau 1. Échelles de cotation de la fréquence d'occurrence, de la probabilité de non-détection et de la gravité de l'effet des modes de défaillance

Échelle Cote

Échelle de cotation de la fréquence d'occurrence du mode de défaillance Une fois par an ou moins

Une fois par 6 mois

Une fois par 4 mois

Une fois par 2 mois

Une fois par mois

Plusieurs fois par mois

Une fois par semaine

Plusieurs fois par semaine

Une fois par jour

Échelle de cotation de la probabilité de non-détection du mode de défaillance survenant dans l'heure suivante

$\begin{array}{ll}\geq 95 \% & 1 \\ 90 \% & 2 \\ 85 \% & 3 \\ 80 \% & 4 \\ 70 \% & 5 \\ 60 \% & 6 \\ 50 \% & 7 \\ 30 \% & 8 \\ 0 \% & 9\end{array}$

Échelle de cotation de la gravité de l'effet du mode de défaillance

Zéro fois la dose ou pas de préjudice

1,25 fois la dose 2

2,5 fois la dose ou préjudice temporaire 4

5 fois la dose 8

10 fois la dose ou préjudice permanent 10

D'abord, l'implantation réalisée dans notre centre s'est accompagnée de la mise en place de feuilles d'ordonnances prérédigées destinées à standardiser les prescriptions des perfusions continues de médicaments. L'Institute for Safe Medication Practices en États-Unis reconnaît les retombées favorables des feuilles d'ordonnances prérédigées sur la réduction du nombre de prescriptions incorrectes ou incomplètes ${ }^{27}$. Notre analyse a révélé que l'utilisation de feuilles d'ordonnances prérédigées a diminué la criticité théorique des erreurs de doses de médicaments en diminuant leur fréquence et en augmentant leur probabilité de non-détection.

Puis la diminution de la criticité peut également être attribuée à la standardisation des concentrations des perfusions continues, qui a éliminé les calculs individualisés en fonction du poids des patients. L'utilisation d'un nombre limité de concentrations standardisées pour la population néonatale fait partie du cadre normatif de la gestion des médicaments par Agrément $\mathrm{Canada}^{28}$. La standardisation des concentrations permet d'éliminer le calcul selon la règle des six, précédemment utilisée dans plusieurs hôpitaux pédiatriques canadiens, et réduit de manière importante la criticité de l'étape de prescription. Il faut noter que la standardisation des concentrations aurait pu s'effectuer sans implantation de pompes intelligentes.

Enfin, la notion de poids de prescription apparaît comme un nouveau mode de défaillance à l'étape de la prescription. Cette notion a été mise en place au sein de notre établissement, car le poids des patients en néonatalogie peut varier rapidement et de manière importante dans le temps. La criticité associée à cette nouvelle pratique est toutefois peu élevée, car la gravité découlant d'une telle erreur reste modérée si l'on considère que le poids de prescription n'excède pas $\pm 25 \%$ du poids réel du patient ${ }^{29}$.

\section{Augmentation de la criticité globale pour l'étape d'administration}

Toutefois, notre analyse a révélé une augmentation de la criticité globale en phase postérieure à l'implantation de pompes intelligentes à l'étape de l'administration de doses de médicaments. Ce résultat s'explique notamment par le fait que la programmation de pompes intelligentes nécessite un plus grand nombre d'étapes que celle des pompes volumétriques précédemment utilisées, ce qui conduit à l'apparition de quatre nouveaux modes de défaillance. Ces derniers concernent la programmation de la pompe intelligente, le non-usage de la bibliothèque de médicaments par omission du personnel soignant, le choix de l'unité de soins où l'administration de la dose se déroule (des paramètres spécifiques peuvent s'appliquer à chaque unité de soins), le choix du bon médicament à partir de la bibliothèque et les erreurs de programmation associées au poids du patient.

Cependant, on a noté une diminution de l'indice de criticité du mode de défaillance associé à l'erreur de débit de dose de médicaments. Ce résultat théorique est cohérent avec le rôle des pompes intelligentes, qui est de sécuriser l'administration intraveineuse des médicaments. Néanmoins, des études ont mis en évidence des erreurs d'administration de doses de médicaments malgré l'utilisation de pompes intelligentes. Ainsi, Husch et al. ont démontré que $97 \%$ des erreurs de débit de doses de médicaments ne sont pas interceptées par les pompes intelligentes, car ces erreurs ne peuvent être détectées par les paramètres saisis dans la pompe (p. ex. il ne s'agit pas d'erreurs de programmation, et les erreurs de doses décrites ne dépassent généralement pas les limites franchissables établies) ${ }^{20}$. Dans l'étude menée par "Russell et al., on a rapporté que jusqu'à $43 \%$ des différences observées entre les ordonnances de médicaments et les paramètres de programmation des pompes intelligentes correspondent à des erreurs de débit de solutés ${ }^{30}$.

\section{Utilité d'une analyse AMDEC}

La tenue d'une étude de type AMDEC antérieure et postérieure à l'implantation de pompes intelligentes nous a été utile pour l'optimisation du circuit du médicament, parce qu'elle 
Tableau 2. Modes de défaillance du processus d'utilisation des perfusions continues, fréquence, probabilité de non-détection, gravité de l'effet et criticité antérieurs et postérieurs à l'implantation

\begin{tabular}{|c|c|c|c|c|c|c|c|c|c|c|}
\hline \multirow[b]{3}{*}{$\begin{array}{l}\text { Étape du processus } \\
\text { d'utilisation des perfusions } \\
\text { continues }\end{array}$} & \multicolumn{5}{|c|}{ Pré-implantation des pompes intelligentes } & \multicolumn{5}{|c|}{ Post-implantation des pompes intelligentes } \\
\hline & \multirow[b]{2}{*}{$\begin{array}{l}\text { Modes de } \\
\text { défaillances }\end{array}$} & \multicolumn{3}{|c|}{ Cotation* } & \multirow[b]{2}{*}{$\begin{array}{l}\text { Indice de } \\
\text { criticitét }\end{array}$} & \multirow[b]{2}{*}{$\begin{array}{c}\text { Modes de } \\
\text { défaillances }\end{array}$} & \multicolumn{3}{|c|}{ Cotation* } & \multirow[b]{2}{*}{$\begin{array}{l}\text { Indice de } \\
\text { criticitét }\end{array}$} \\
\hline & & $\mathbf{F}$ & $\mathbf{P}$ & G & & & $\mathbf{F}$ & $\mathbf{P}$ & $\mathbf{G}$ & \\
\hline \multirow[t]{8}{*}{ Prescription } & Erreur de dose & 2 & 2 & 10 & 40 & Erreur de dose & 1 & 1 & 10 & 10 \\
\hline & Erreur de débit & 2 & 2 & 10 & 40 & $\begin{array}{l}\text { Erreur dans le } \\
\text { calcul du débit }\end{array}$ & 1 & 1 & 10 & 10 \\
\hline & $\begin{array}{l}\text { Erreur de calcul } \\
\text { de concentration }\end{array}$ & 7 & 2 & 10 & 140 & Ne pas utiliser FOPR & R 7 & 1 & 10 & 70 \\
\hline & $\begin{array}{l}\text { Solution trop } \\
\text { concentrée } \\
\text { (osmolarité) }\end{array}$ & 3 & 8 & 10 & 240 & $\begin{array}{l}\text { Choix de la } \\
\text { mauvaise FOPR }\end{array}$ & 1 & 1 & 10 & 10 \\
\hline & $\begin{array}{l}\text { Erreur dans le } \\
\text { choix du diluant }\end{array}$ & 5 & 2 & 2 & 20 & $\begin{array}{l}\text { Erreur dans le } \\
\text { choix du diluant }\end{array}$ & 5 & 1 & 2 & 10 \\
\hline & $\begin{array}{l}\text { Choix de la } \\
\text { mauvaise } \\
\text { concentration (ap } \\
\text { liquidien élevé) }\end{array}$ & 3 & 2 & 4 & 24 & $\begin{array}{l}\text { Choix de la } \\
\text { mauvaise } \\
\text { concentration } \\
\text { (apport liquidien } \\
\text { élevé) }\end{array}$ & 6 & 1 & 4 & 24 \\
\hline & & & & & & $\begin{array}{l}\text { Erreur de poids } \\
\text { de prescription }\end{array}$ & 5 & 4 & 2 & 40 \\
\hline & Criticité globale & NA & NA & NA & 504 & Criticité globale & NA & NA & NA & 174 \\
\hline \multirow[t]{4}{*}{ Préparation } & $\begin{array}{l}\text { Erreur de calcul } \\
\text { pour ramener le } \\
\text { volume à } 50 \mathrm{~mL}\end{array}$ & 2 & 1 & 10 & 20 & $\begin{array}{l}\text { Erreur dans le } \\
\text { volume du } \\
\text { médicament } \\
\text { prélevé }\end{array}$ & 3 & 1 & 10 & 30 \\
\hline & $\begin{array}{l}\text { Erreur dans le } \\
\text { volume du } \\
\text { médicament } \\
\text { prélevé }\end{array}$ & 5 & 1 & 10 & 50 & $\begin{array}{l}\text { Non-utilisation } \\
\text { des outils }\end{array}$ & 5 & 1 & 10 & 50 \\
\hline & $\begin{array}{l}\text { Non-utilisation } \\
\text { des outils }\end{array}$ & 5 & 1 & 10 & 50 & $\begin{array}{l}\text { Erreur dans le } \\
\text { choix de la fiche } \\
\text { recette de } \\
\text { préparation }\end{array}$ & 3 & 1 & 10 & 30 \\
\hline & Criticité globale & NA & NA & NA & 120 & Criticité globale & NA & NA & NA & 110 \\
\hline \multirow[t]{7}{*}{ Administration } & Erreur de débit & 7 & 4 & 10 & 280 & Erreur de débit & 7 & 1 & 10 & 70 \\
\hline & $\begin{array}{l}\text { Erreur de site } \\
\text { d'administration }\end{array}$ & 2 & 8 & 10 & 160 & $\begin{array}{l}\text { Erreur de site } \\
\text { d'administration }\end{array}$ & 2 & 6 & 10 & 120 \\
\hline & & & & & & $\begin{array}{l}\text { Non-utilisation de } \\
\text { la bibliothèque } \\
\text { de la pompe }\end{array}$ & 7 & 1 & 10 & 70 \\
\hline & & & & & & $\begin{array}{l}\text { Erreur dans le choix } \\
\text { de l'unité de soins }\end{array}$ & $\times 1$ & 8 & 10 & 80 \\
\hline & & & & & & $\begin{array}{l}\text { Erreur dans le } \\
\text { choix de l'entrée } \\
\text { du médicament et } \\
\text { la concentration }\end{array}$ & 5 & 1 & 10 & 50 \\
\hline & & & & & & Erreur de poids & 7 & 2 & 10 & 140 \\
\hline & Criticité globale & NA & NA & NA & 440 & Criticité globale & NA & NA & NA & 530 \\
\hline Total & Criticité globale & NA & NA & NA & 1064 & Criticité globale & NA & NA & NA & 814 \\
\hline
\end{tabular}


a permis de réunir des cliniciens de différentes formations, de déterminer les modes de défaillance et d'en parler, et de réfléchir à des actions correctrices. Cette réflexion entourant les pompes intelligentes en néonatologie a notamment permis de détecter de nouveaux modes de défaillance, et cette démarche a contribué à la mise en place de plusieurs mesures correctrices applicables à l'ensemble de notre établissement (p. ex. exigence d'écrire le poids de prescription applicable à chaque ordonnance de perfusion de médicaments, formation répétée afin de limiter le recours au mode de perfusion ne tenant pas compte de la bibliothèque ou " hors bibliothèque " et mise à jour d'un portail Web regroupant l'ensemble des documents et des données destinés au bon usage des pompes). De plus, la réflexion menée a contribué à faciliter l'analyse des rapports d'incidents et d'accidents en rendant les cliniciens conscients des difficultés inhérentes à l'utilisation d'une nouvelle technologie. Enfin, la réflexion a permis une utilisation plus sécuritaire et responsable de cette nouvelle technologie.

\section{Limites}

Cette étude comporte des limites. Nous avons étudié uniquement les pratiques applicables au circuit du médicament du secteur de néonatologie. Notons que l'introduction des ordonnances prérédigées visant à standardiser les concentrations des médicaments administrés en perfusion a pu, indépendamment de l'utilisation de pompes intelligentes, diminuer la criticité.

\section{CONCLUSIONS}

Lanalyse AMDEC a démontré une réduction globale du risque théorique lié à l'utilisation des perfusions intraveineuses continues, processus comportant des risques élevés d'erreurs médicamenteuses. La mise en place de prescriptions et de concentrations standardisées a diminué le risque théorique de l'étape de prescription et de préparation qui sont des étapes critiques du fait de l'individualisation des doses des médicaments en néonatalogie. On a démontré en revanche une augmentation de la criticité globale en phase postérieure à l'implantation pour l'étape d'administration.

\section{Références}

1. Kaushal R, Bates DW, Landrigan C, McKenna KJ, Clapp MD, Federico F, et al. Medication errors and adverse drug events in pediatric inpatients. JAMA. 2001;285(16):2114-20.

2. Hicks RW, Becker SC. An overview of intravenous-related medication administration errors as reported to MEDMARX, a national medication error-reporting program. J Infus Nurs. 2006;29(1):20-7.

3. Top 10 health technology hazards for 2013 are named. OR Manager. 2013;29(2):16-9.

4. Conroy S, Sweis D, Planner C, Yeung V, Collier J, Haines L, et al. Interventions to reduce dosing errors in children: a systematic review of the literature. Drug Saf. 2007;30(12):1111-25.

5. Simpson JH, Lynch R, Grant J, Alroomi L. Reducing medication errors in the neonatal intensive care unit. Arch Dis Child Fetal Neonatal Ed. 2004;89(6):F480-2.

6. Chedoe I, Molendijk HA, Dittrich ST, Jansman FG, Harting JW, Brouwers $\mathrm{JR}$, et al. Incidence and nature of medication errors in neonatal intensive care with strategies to improve safety: a review of the current literature. Drug Saf. 2007;30(6):503-13.
7. Oren E, Shaffer ER, Guglielmo BJ. Impact of emerging technologies on medication errors and adverse drug events. Am J Health Syst Pharm. 2003;60(14):1447-58.

8. Wong IC, Wong LY, Cranswick NE. Minimising medication errors in children. Arch Dis Child. 2009;94(2):161-4.

9. Venkatraman R, Durai R. Errors in administration: how can they be minimised? J Perioper Pract. 2008;18(6):249-53.

10. Benoit E, Beney J. Les nouvelles technologies permettent-elles de réduire les erreurs médicamenteuses en soins intensifs adultes? J Pharm Belg. $2011 ;(3): 82-91$.

11. Gonzales K. Medication administration errors and the pediatric population: a systematic search of the literature. J Pediatr Nurs. 2010;25(6):555-65.

12. Manias E, Williams A, Liew D. Interventions to reduce medication errors in adult intensive care: a systematic review. $\mathrm{Br} J$ Clin Pharmacol. 2012;74(3):411-23.

13. Cour M, Hernu R, Bénet T, Robert JM, Regad D, Chabert B, et al. Benefits of smart pumps for automated changeovers of vasoactive drug infusion pumps: a quasi-experimental study. Br J Anaesth. 2013;111(5):818-24.

14. Prewitt J, Schneider S, Horvath M, Hammond J, Jackson J, Ginsberg B. PCA safety data review after clinical decision support and smart pump technology implementation. J Patient Saf. 2013;9(2):103-9.

15. Which smart pumps are smartest? Health Devices. 2012;41(12):378-91.

16. Kastrup M, Balzer F, Volk T, Spies C. Analysis of event logs from syringe pumps: a retrospective pilot study to assess possible effects of syringe pumps on safety in a university hospital critical care unit in Germany. Drug Saf. 2012;35(7):563-74.

17. Tran M, Ciarkowski S, Wagner D, Stevenson JG. A case study on the safety impact of implementing smart patient-controlled analgesic pumps at a tertiary care academic medical center. Jt Comm J Qual Patient Saf. 2012; 38(3):112-9.

18. Larsen GY, Parker HB, Cash J, O'Connell M, Grant MC. Standard drug concentrations and smart-pump technology reduce continuous-medicationinfusion errors in pediatric patients. Pediatrics. 2005;116(1):e21-5.

19. Rothschild JM, Keohane CA, Cook EF, Orav EJ, Burdick E, Thompson S, et al. A controlled trial of smart infusion pumps to improve medication safety in critically ill patients. Crit Care Med. 2005;33(3):533-40.

20. Husch M, Sullivan C, Rooney D, Barnard C, Fotis M, Clarke J, et al. Insights from the sharp end of intravenous medication errors: implications for infusion pump technology. Qual Saf Health Care. 2005;14(2):80-6.

21. Failure modes and effects analysis (FMEA) tool. Cambridge (MA) : Institute for Healthcare Improvement; 2014. Publié au : www.ihi.org. Consulté le 10 octobre 2014.

22. Adachi W, Lodolce AE. Use of failure mode and effects analysis in improving the safety of intravenous drug administration. Am J Health Syst Pharm. 2005;62(9):917-20.

23. DeRosier J, Stalhandske E, Bagian JP, Nudell T. Using health care failure mode and effect analysis: the VA National Center for Patient Safety's prospective risk analysis system. Jt Comm J Qual Improv. 2002;28(5):248-67.

24. Fassett WE. Key performance outcomes of patient safety curricula: root cause analysis, failure mode and effects analysis, and structured communications skills. Am J Pharm Educ. 2011;75(8):164.

25. Failure mode and effects analysis (FMEA). Toronto (ON): Institute for Safe Medication Practices Canada; 2014. Publié au : https://www.ismpcanada.org/fmea.htm. Consulté le 10 octobre 2014.

26. ISMP list of high-alert medications in acute care settings. Horsham (PA): Institute for Safe Medication Practices; 2014. Publié au www.ismp.org/ Tools/highalertmedications.pdf. Consulté le 10 octobre 2014.

27. ISMP's guidelines for standard order sets. Horsham (PA): Institute for Safe Medication Practices; 2010. Publié au : www.ismp.org/tools/guidelines/ standardordersets.pdf. Consulté le 10 octobre 2014.

28. Medication management standards. Ottawa $(\mathrm{ON})$ : Accreditation Canada; 2013. Disponible pour achat au : www.accreditation.ca/medicationmanagement-standards

29. Tourel J, Delage E, Lebel D, Litalien C, Duval S, Lacroix A, et al. Smart pump use in pediatric patients. Am J Health Syst Pharm. 2012;69(19):1628-9.

30. Russell RA, Murkowski K, Scanlon MC. Discrepancies between medication orders and infusion pump programming in a paediatric intensive care unit. Qual Saf Health Care. 2010;19 Suppl 3:i31-5.

Emmanuelle Delage, D. Pharm., est assistante de recherche à l'Unité de recherche en pratique pharmaceutique, Centre hospitalier universitaire Sainte-Justine, Montréal, Québec. 
Julien Tourel, D. Pharm., est assistant de recherche à l'Unité de recherche en pratique pharmaceutique, Centre hospitalier universitaire SainteJustine, Montréal, Québec.

Brigitte Martin, B. Pharm., M. Sc., est pharmacienne, Département de pharmacie, Centre hospitalier universitaire Sainte-Justine, Montréal, Québec.

Aurélie Guérin est candidate au D. Pharm. et assistante de recherche à I'Unité de recherche en pratique pharmaceutique, Centre hospitalier universitaire Sainte-Justine, Montréal, Québec. Elle est aussi interne en pharmacie, Université Paris Sud XI, Paris, France.

Ahmed Moussa, M. D., FRCPC, FAAP, est néonatalogiste, Département de néonatalogie, Centre hospitalier universitaire Sainte-Justine, et professeur adjoint de clinique, Université de Montréal, Montréal, Québec.

Annie Lacroix, B.Sc. Inf, M. Sc., IPSNN, est chef des processus administratifs en soins infirmiers, Département de néonatalogie, Centre hospitalier universitaire Sainte-Justine, Montréal, Québec.
Denis Lebel, B. Pharm., M. Sc., FCSHP, est adjoint, Département de pharmacie et Unité de recherche en pratique pharmaceutique, Centre hospitalier universitaire Sainte-Justine, Montréal, Québec.

Jean-François Bussières, B. Pharm., M. Sc., FCSHP, est chef, Département de pharmacie et Unité de recherche en pratique pharmaceutique, Centre hospitalier universitaire Sainte-Justine, et professeur titulaire de clinique, Faculté de pharmacie, Université de Montréal, Montréal, Québec. Intérêts concurrents : Aucun déclaré.

Adresse de correspondance :

Jean-François Bussières

Département de pharmacie

Centre hospitalier universitaire Sainte-Justine

3175, chemin de la Côte Sainte-Catherine

Montréal QC H3T 1C5

Courriel : jf.bussieres@ssss.gouv.qc.ca

Financement : Aucun.

\title{
$\square$ Canadian Society of Hospital Pharmacists Société canadienne des pharmaciens d'hôpitaux Membership Year 2015/2016 (July 1, 2015 - June 30, 2016)
}

\author{
Are you a Member or Supporter of the Canadian Society of Hospital Pharmacists?
}

CSHP has celebrated 67 years of inspiration and leadership among a growing network of over 3000 pharmacy colleagues. When you join CSHP, you have access to resources and opportunities that will enhance your career and help influence healthcare in Canada. Thank you for your participation in CSHP 2015, an initiative challenging all pharmacists to improve patient medication outcomes and safety through advancing pharmacy practice excellence by the year 2015 .

$$
\text { Renew or join today! }
$$

Membership Enquiries: Please contact Membership Services

Tel.: (613) 736-9733 Ext. 222 | Fax: (613) 736-5660 | Email: membershipservices@cshp.ca www.cshp.ca 